\title{
High-Frequency Conductivity Plateau and Ionic Hopping Processes in a Ternary Lithium Borate Glass
}

\author{
C. Cramer ${ }^{a}$, K. Funke ${ }^{a}$, T. Saatkamp ${ }^{a}$, D. Wilmer ${ }^{a}$, and M. D. Ingram ${ }^{b}$ \\ a Institut für Physikalische Chemie, Schlossplatz 4/7, D-48149 Münster \\ b Department of Chemistry, University of Aberdeen, Meston Walk, Aberdeen, AB9 2UE, \\ Scotland, UK
}

Z. Naturforsch. 50a, 613-623 (1995); received November 8, 1995

\section{Dedicated to Prof. W. Müller-Warmuth on the occasion of his 65th birthday}

\begin{abstract}
Complete conductivity spectra of a lithium ion conducting glass of composition $\mathrm{B}_{2} \mathrm{O}_{3}-0.56 \mathrm{Li}_{2} \mathrm{O}-$ $0.45 \mathrm{LiBr}$ have been taken at twelve temperatures from $173 \mathrm{~K}$ to $573 \mathrm{~K}$, covering the frequency range from a few Hertz up to about $50 \mathrm{THz}$. For the first time, a separation of the hopping and vibrational contributions to the spectra has been performed. The resulting frequency dependent hopping conductivity displays a high-frequency plateau similar to those known to exist in crystalline solid electrolytes like $\mathrm{RbAg}_{4} \mathrm{I}_{5}$ and $\mathrm{Na}-\beta$-alumina. In the dispersive regime of the conductivity, there is an increase of the apparent power-law exponent from $p=0.6$ to $q=1$. The spectra are evaluated by combined application of the jump relaxation model and the dynamic structure model. Besides the translational hopping of the lithium ions via the preferred $\bar{A}$ sites, more localized kinds of motion are also detected, viz., hops into the less favoured $\bar{C}$ sites (and mostly back again) as well as smaller displacements of the ions within their local environment.
\end{abstract}

\section{Introduction: Hopping Conductivities of Crystalline and Glassy Solid Electrolytes}

Measurement of dynamic conductivities, $\sigma(\omega)$, of solid electrolytes up to far-infrared frequencies is a valuable tool for studying the ion dynamics in these materials. In Sect. II we will present conductivity spectra of a lithium ion conducting lithium bromideborate glass obtained at various temperatures. To provide a basis for a discussion of the data, let us first mention a few characteristics of conductivity spectra of crystalline and glassy electrolytes.

In both crystalline and glassy ionic conductors, the universal Jonscher power law of the conductivity [1] is well fulfilled as long as the angular frequency, $\omega$, is sufficiently low:

$$
\sigma(\omega) \approx \sigma(0) \cdot\left[1+\left(\omega t_{2}\right)^{p}\right], \quad 0<p<1 .
$$

Here, $\sigma(\omega)$ and $\sigma(0)$ denote the frequency dependent and the dc conductivity, respectively, while $1 / t_{2}$ marks the onset of the dispersion on the angular-frequency scale.

\footnotetext{
* Present address: Argonne National Laboratory, Materials Science Division, Building 223, 9700 South Cass Avenue, Argonne, IL 60439, USA.

Reprint requests to Prof. Dr. K. Funke.
}

In the last few years, another new universality has become apparent $[2,3]$ which is also common to crystalline and glassy electrolytes. At low temperatures, typically about $100 \mathrm{~K}$ and below, the electrical conductivity does not seem to fall below a certain baseline which remains constant when the temperature is further decreased and which varies exactly with the first power of frequency. To date, a satisfactory explanation does not exist.

In the activated-hopping temperature regime, the low-frequency part of $\sigma(\omega)$ is always well described by (1) in both crystals and glasses. At higher frequencies, however, there are major differences:

(i) In crystalline fast ion conductors like $\operatorname{RbAg}_{4} \mathrm{I}_{5}[4,5]$ and $\mathrm{Na}-\beta$-alumina [6] the power-law behaviour of (1) eventually merges into a high-frequency plateau which is observed at millimetre-wave frequencies, just before the conductivity is dominated by vibrational modes of motion.

The overall shape of hopping-conductivity spectra like that of Fig. 1 a (below about $100 \mathrm{GHz}$ ) is well reproduced by the jump relaxation model [7-12], and also by Ngai's coupling model [12-15]. The jump relaxation model accounts for Coulomb interactions between the structurally disordered mobile ions. It predicts the frequent occurrence of forward-backward hopping sequences. A hop followed by a correlated 
a)

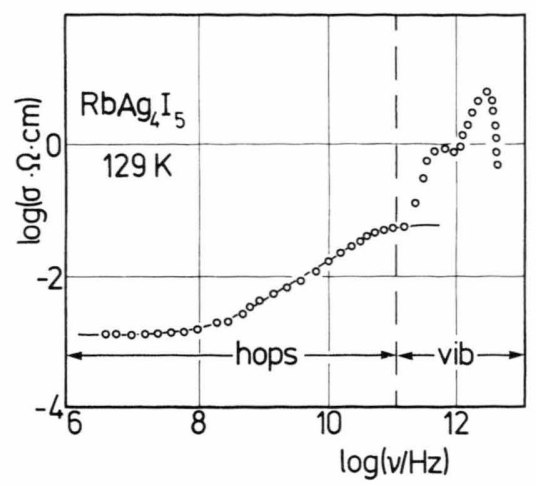

b)

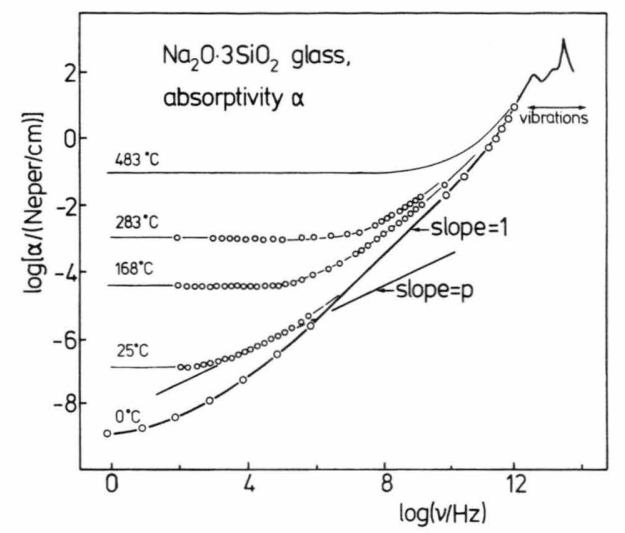

Fig. 1. a) Dynamic conductivity of the crystalline solid electrolyte $\mathrm{RbAg}_{4} \mathrm{I}_{5}$ at $129 \mathrm{~K}$ after [4] and b) absorptivity/conductivity spectra of $\mathrm{Na}_{2} \mathrm{O}-3 \mathrm{SiO}_{2}$ glass at various temperatures, after [16] and [18]. 1 Neper $\mathrm{cm}^{-1}$ corresponds to $3 \cdot 10^{-3} \Omega^{-1} \mathrm{~cm}^{-1}$.

backward hop is said to be unsuccessful; it does not contribute to the dc conductivity. At high frequencies, however, every hop is recorded and thus contributes to the high-frequency plateau.

It is important to note that a successful hopping requires site relaxation. In the model, this is equivalent to a shifting of the Coulomb cage from the former to the present position of the ion. The process is supposed to be entirely due to the hopping motion of the other ions in the system.

(ii) In glassy electrolytes, the conductivity spectrum is rather different in appearance. The apparent powerlaw exponent seems to increase with increasing frequency and there is indication of a linear behaviour [16-18],

$$
\sigma(\omega) \propto \omega^{1.0},
$$

at intermediate frequencies. At still higher frequencies, exponents have occasionally been found to exceed one $[19,20]$, though experimental data are scarce.

In glass, the existence of a high-frequency plateau has never been reported. Therefore, it is a challenging question to find out how the hopping conductivity of glass merges into the vibrational one. In section II we will show that the extrapolated vibrational contribution can be subtracted out of the entire conductivity, resulting in hopping-conductivity spectra that $d o$ display high-frequency plateaux. In Sects. III to V, the main features of our spectra will then be traced back to their generic dynamic processes. This will be done by a combined application of the jump relaxation model [7] and the dynamic structure model [21 -25].

\section{Complete Conductivity Spectra of a Ternary Lithium-Borate glass}

Complete conductivity spectra of a lithium ion conducting glass of composition $\mathrm{B}_{2} \mathrm{O}_{3}-0.56 \mathrm{Li}_{2} \mathrm{O}-0.45$ $\mathrm{LiBr}$ have been taken at twelve temperatures ranging from $173 \mathrm{~K}$ to $573 \mathrm{~K}$. Various experimental techniques have been employed in order to cover the unusually wide frequency range from a few Hertz up to $50 \mathrm{THz}$. These include impedance, radio, microwave, and FTIR spectroscopy. Details are described in $[4,26]$.

Figures $2 \mathrm{a}$ and $2 \mathrm{~b}$ are $\log -\log$ representations of the ionic conductivity versus frequency showing complete spectra at $573 \mathrm{~K}$ and $323 \mathrm{~K}$, respectively. At first glance it is evident that the overall shape of the spectra is in agreement with the features described in section I. Up to about $1 \mathrm{THz}$, the power-law exponent seems to increase continuously with increasing frequency. In particular, a high-frequency plateau is not visible.

In the far infrared, the conductivity spectra are found to vary with frequency as $\omega^{2}$, the temperature dependence being rather small. These data can be regarded as the low-frequency flank of the lowestlying vibrational mode. The vibrational contribution to the conductivity is now easily extrapolated to lower frequencies and taken out of the total spectra. The resulting spectra are then interpreted in terms of the hopping motion of the mobile ions.

Figures $3 \mathrm{a}$ and $3 \mathrm{~b}$ show the resulting hopping-conductivity spectra (+) as well as the total conductivity ones. The most exciting feature is the appearance of a high-frequency plateau. This is more clearly seen 

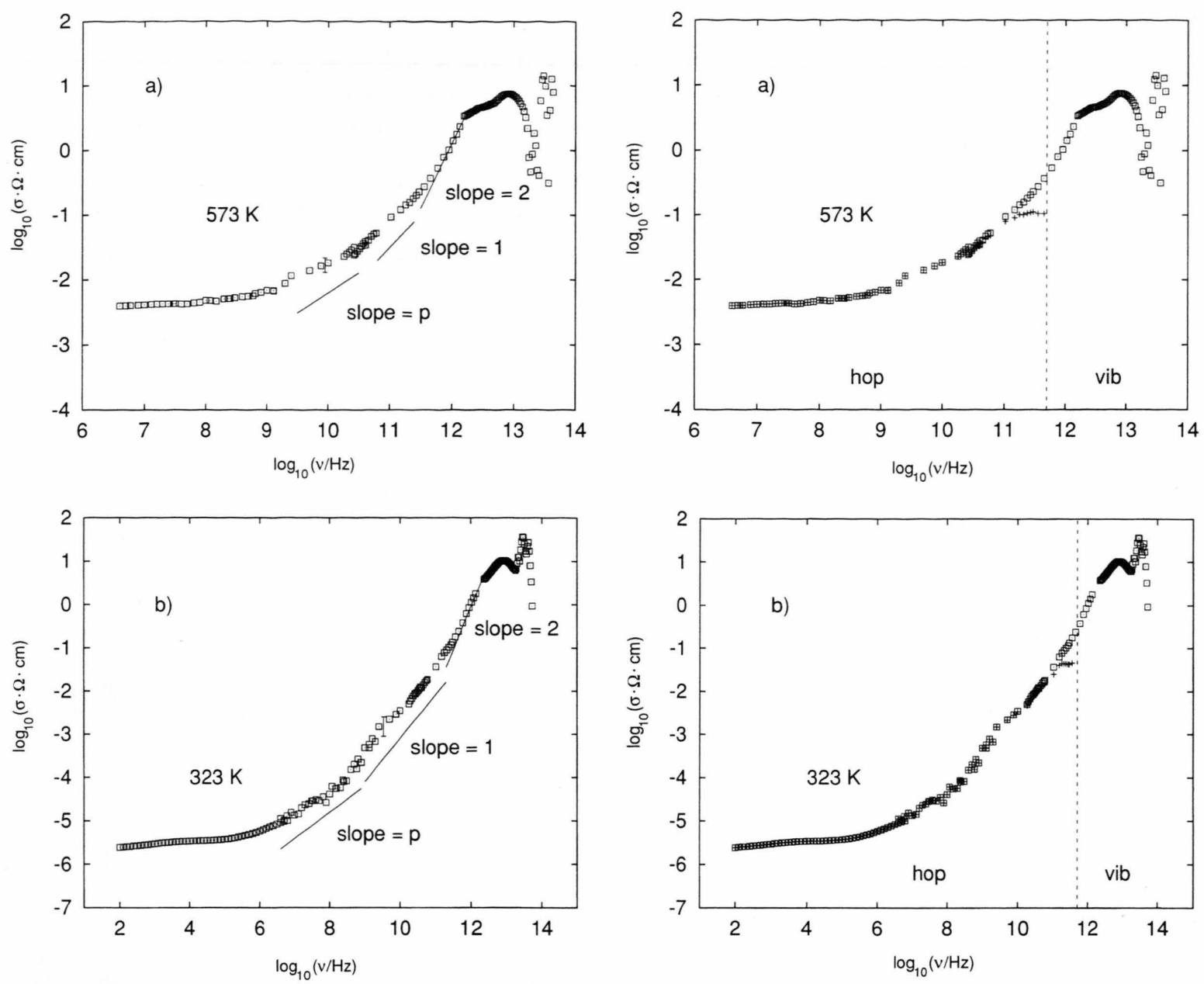

Fig. 2. Complete conductivity spectra of $\mathrm{B}_{2} \mathrm{O}_{3}-0.56 \mathrm{Li}_{2} \mathrm{O}$ $0.45 \mathrm{LiBr}$ glass at a) $573 \mathrm{~K}$ and b) $323 \mathrm{~K}$. The error bars indicate the largest possible error over the entire frequency range.

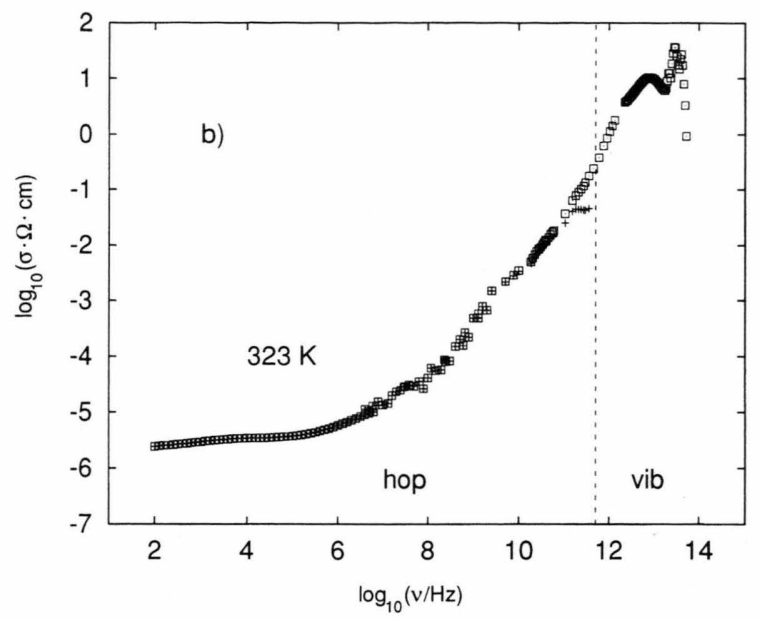

Fig. 3. Total experimental conductivity spectra (ㅁ) and spectra obtained after subtracting vibrational contributions out of them $(+)$, of glassy $\mathrm{B}_{2} \mathrm{O}_{3}-0.56 \mathrm{Li}_{2} \mathrm{O}-0.45 \mathrm{LiBr}$ at a) $573 \mathrm{~K}$ and b) $323 \mathrm{~K}$.

when the data are looked at more closely between $10^{10}$ and $10^{12} \mathrm{~Hz}$, see Figs. $4 \mathrm{a}$ and $4 \mathrm{~b}$. At about $10^{11} \mathrm{~Hz}$ the data merge into their plateau values.

With regard to their overall shape, the spectra $(+)$ of Fig. 3 do, indeed, resemble those of crystalline electrolytes, cf. Figure $1 \mathrm{a}$. They are also in broad agreement with the predictions of the coupling and jump relaxation models. It has often been claimed that these models do not apply for glasses, because they predict a high-frequency plateau which has never been observed. This argument is now invalidated. The reason for high-frequency plateaux not being directly visible in experimental conductivity spectra of glass is the

existence of a broad vibrational contribution which swamps the high-frequency part of the hopping conductivity.

Complementing the spectra (+) of Fig. 3 we present additional ones in Figs. 5 and 6. The solid lines result from a simple model construction which will be described in Sects. III to V.

At low temperatures, the new universality behaviour is clearly encountered. In fact, the conductivities seem to converge to a base-line which varies with frequency as $\omega^{1.0}$. Plots of $\log \sigma$ vs. $1 / T$ at different frequencies lead us to believe that this base-line is well approximated by the dashed line in Figure 6. Its contribution 

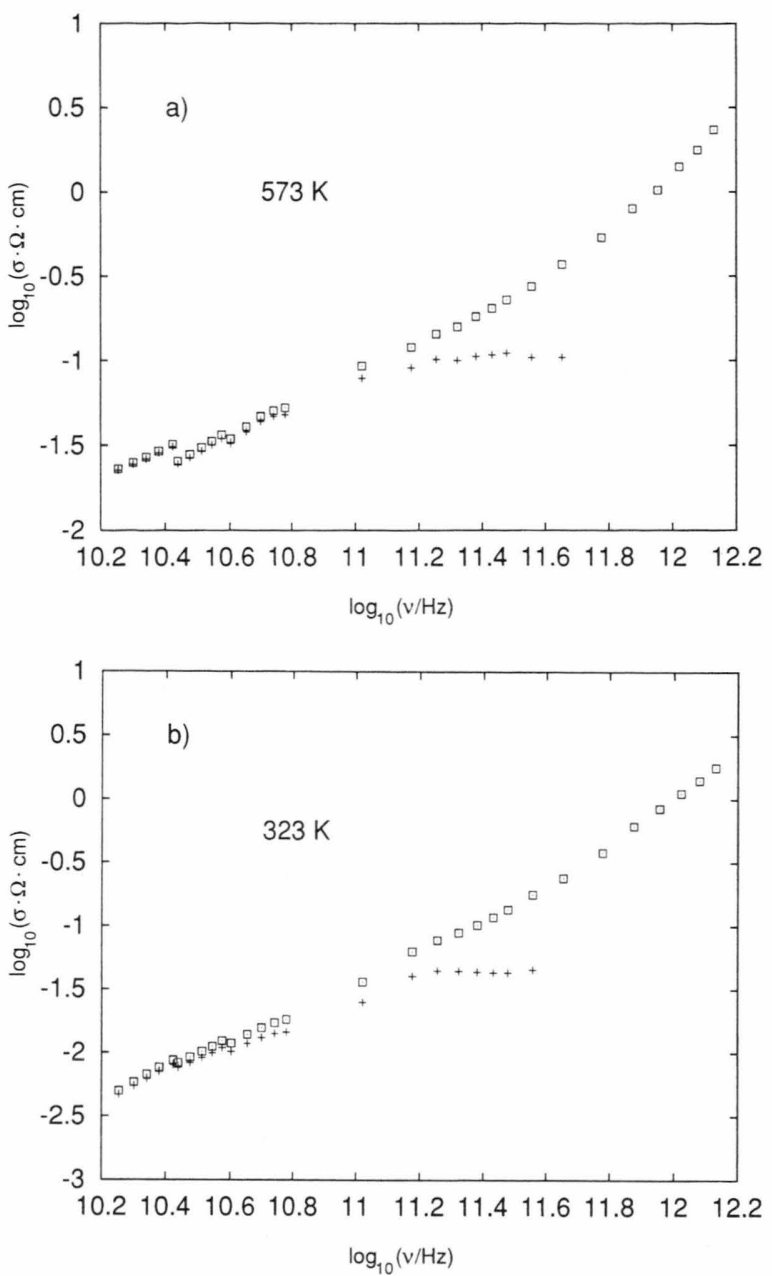

Fig. 4. Representations of the high-frequency plateau regions of Fig. 3 at the same temperatures.

to the overall conductivity spectra is, however, important only at relatively low temperatures.

At $173 \mathrm{~K}$, small thermally activated deviations from this base-line are already observed at both low and high frequencies. With increasing temperature, these activated contributions to $\sigma(\omega)$ are found to develop in different ways.

The activated low-frequency component is the universal Jonscher-type part of the spectra, see (1), including a dc conductivity which obeys an Arrhenius law:

$$
\sigma(0) \cdot T=\sigma_{\text {hop }}(0) \cdot T=A_{\mathrm{o}} \cdot \exp \left(-E_{0} / k T\right) .
$$

Figure 7 is an Arrhenius plot of the dc conductivity. The values of the parameters are $E_{0}=k \cdot 5670 \mathrm{~K}$ $(0.49 \mathrm{eV})$ and $A_{0}=4.2 \cdot 10^{4} \Omega^{-1} \mathrm{~cm}^{-1} \mathrm{~K}$.
In contrast to $\sigma(0)$, the activated high-frequencyplateau conductivity does not obey a simple Arrhenius law. The same holds true for hopping conductivities obtained at microwave frequencies, see Figure 8. There seem to be two Arrhenius-type contributions to the high-frequency hopping conductivity, their activation energies being about $0.22 \mathrm{eV}$ and $0.04 \mathrm{eV}$, respectively, see Section V. The solid lines of Fig. 8 result from the same model construction as those of Figs. 5 and 6. An outline of this model construction will be given in the following sections.

\section{Spectra and Model Conceptions}

In the high-frequency-plateau regime, the sign of the applied electric field changes so fast that every hop or displacement of an ion is recorded and contributes to the conductivity. At slightly higher FIR frequencies, the period of the field is already comparable with the duration of an individual hop, and this implies the breakdown of the very concept of hopping conductivity. At the same time, the subtraction described in Sect. II is no longer feasible, if the frequency exceeds about $400 \mathrm{GHz}$.

All kinds of hops and displacements of the ions in the glass contribute to the plateau conductivity. For a more detailed specification of these contributions and of their generic hopping processes it is useful to consider the temperature dependence of the plateau conductivity, see Fig. 8, as well as the hopping conductivity observed at lower frequencies, see Figs. 3, 5, 6.

A remarkable feature shows up in Fig. 9 giving the particular example of the $373 \mathrm{~K}$ hopping-conductivity spectrum. At all temperatures, like in Fig. 9, the lowfrequency part of the conductivity is nicely fitted by (1), with $p=0.6$. On the other hand, the high-frequency part of the hopping conductivity is at all temperatures close to

$$
\sigma_{\mathrm{hop}}=\sigma(\infty) \cdot\left[1+\left(\omega t_{1}\right)^{-1}\right]^{-q} ; \quad q>1 .
$$

In (4), $\sigma(\infty)$ and $t_{1}$ denote the plateau value of $\sigma_{\text {hop }}$ and the inverse angular frequency at the crossover from the power-law behaviour into the plateau conductivity. Concerning the exponent $q$, the best fit is

$$
q=1.3 \text {. }
$$

Both limiting cases are reminiscent of the jump relaxation model which predicts [7]

$$
\sigma_{\text {hop }}=\sigma(0)+[\sigma(\infty)-\sigma(0)] \cdot\left[1+\left(\omega t_{1}\right)^{-1}\right]^{-p} .
$$



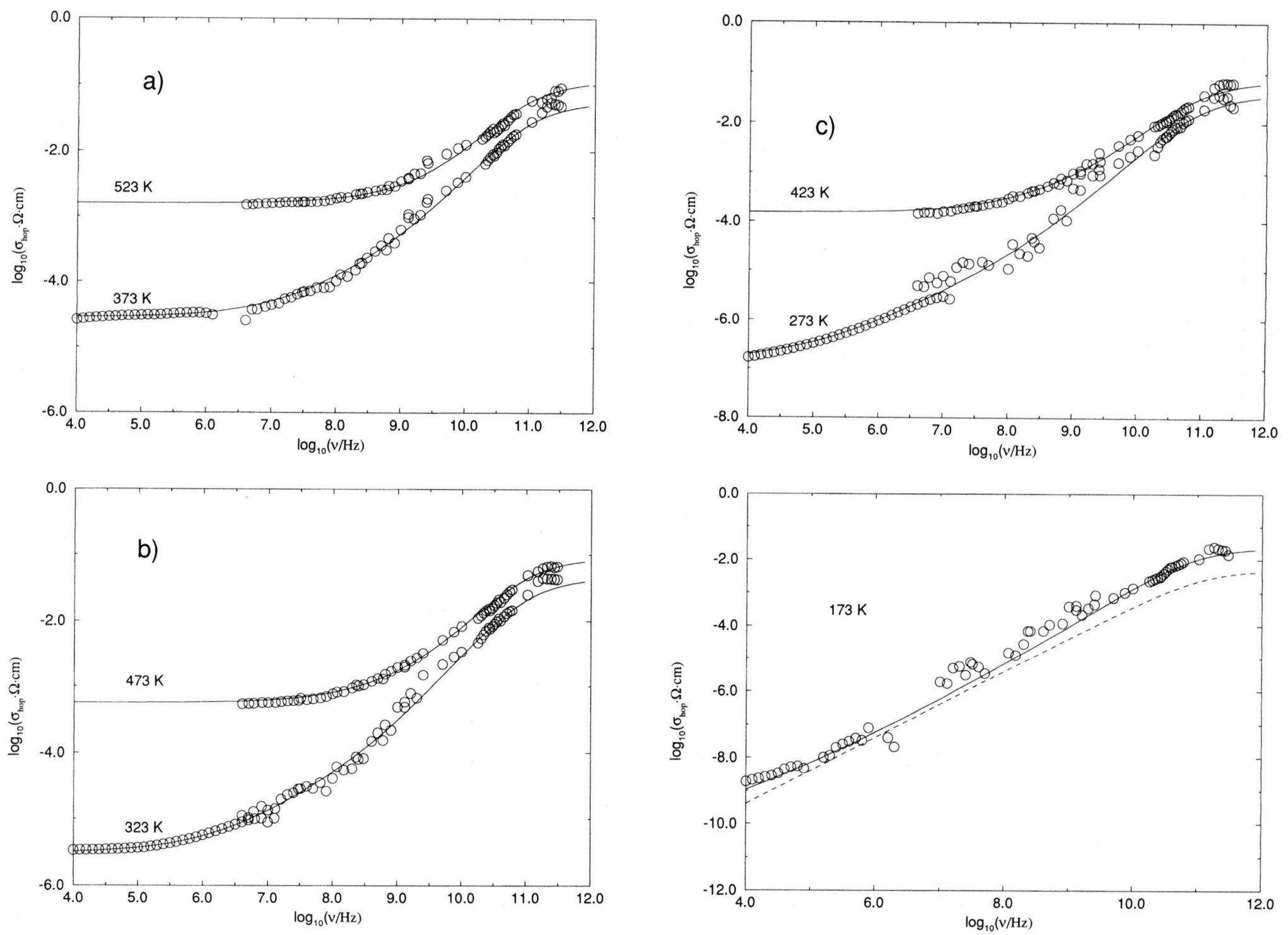

Fig. 5. Hopping conductivity spectra of glassy $\mathrm{B}_{2} \mathrm{O}_{3}-0.56 \mathrm{Li}_{2} \mathrm{O}-0.45 \mathrm{LiBr}$ (o). Solid lines result from our model construction, see sections III to V. a) $523 \mathrm{~K}$ and $373 \mathrm{~K}$, b) $473 \mathrm{~K}$ and $323 \mathrm{~K}$, c) $423 \mathrm{~K}$ and $273 \mathrm{~K}$.

Fig. 6. Hopping conductivity spectra of glassy $\mathrm{B}_{2} \mathrm{O}_{3}-0.56 \mathrm{Li} \mathrm{O}-0.45 \mathrm{LiB}$ at $173 \mathrm{~K}$. Solid line: model construction, see sections III to V. Dashed line: $\omega^{1.0}$ base-line. 


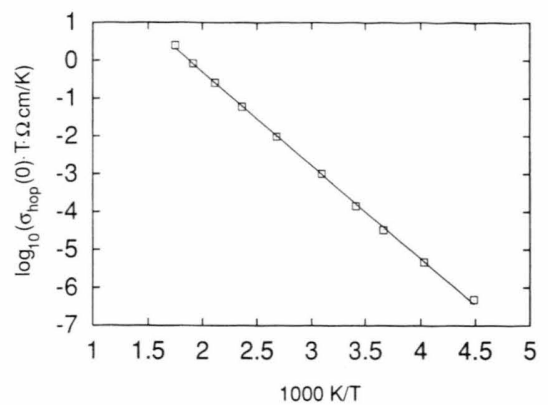

Fig. 7. Arrhenius plot of the dc conductivity of $\mathrm{B}_{2} \mathrm{O}_{3}-$ $0.56 \mathrm{Li}_{2} \mathrm{O}-0.45 \mathrm{LiBr}$ glass.

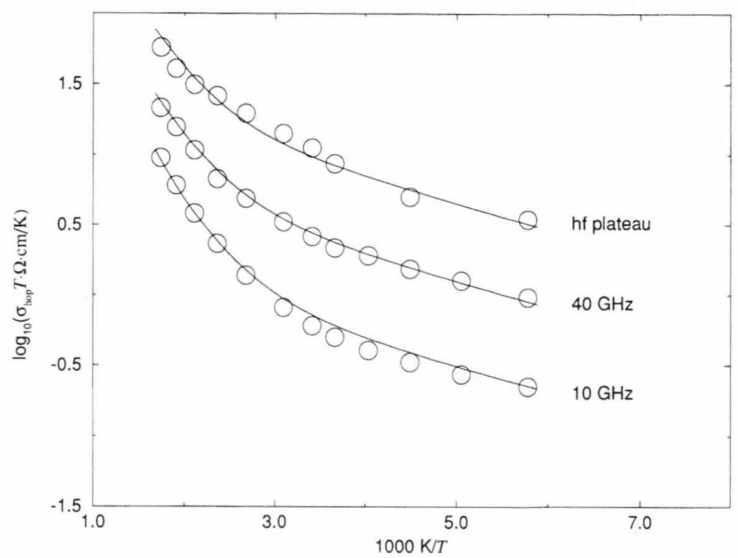

Fig. 8. Arrhenius type representation of hopping conductivities $\sigma_{\text {hop }}(0)$ of glassy $\mathrm{B}_{2} \mathrm{O}_{3}-0.56 \mathrm{Li}_{2} \mathrm{O}-0.45 \mathrm{LiBr}$ at different frequencies. Solid line: model construction as explained in Sects. III to V.

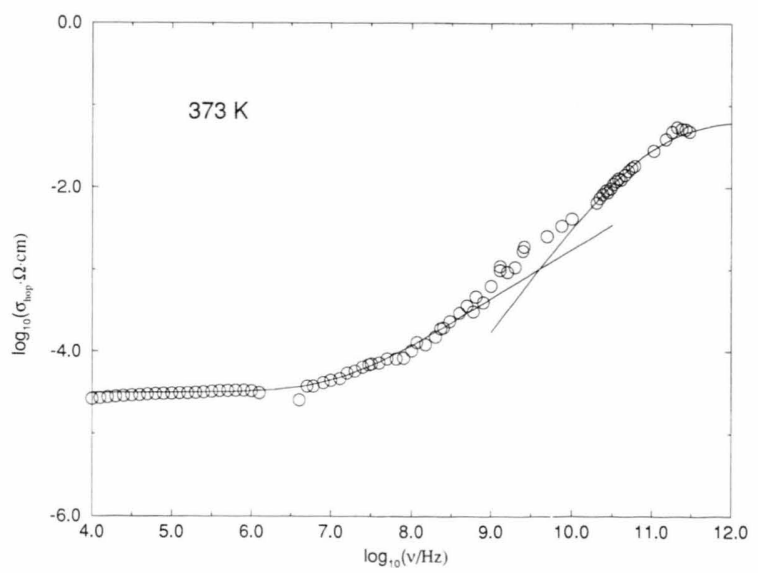

Fig. 9. Hopping conductivity spectrum of $\mathrm{B}_{2} \mathrm{O}_{3}-0.56 \mathrm{Li}_{2} \mathrm{O}-$ $0.45 \mathrm{LiBr}$ glass at $373 \mathrm{~K}(\mathrm{O})$. Solid lines calculated via (1) (low frequency regime, $p=0.6$ ) and (4) (high frequency regime, $q=1.3$ ).
The only difference is that we now have $q \neq p$ and $q>1$, which is impossible for a system that fulfils the assumptions made in that model. The two basic assumptions of the jump relaxation model are:

(i) There is only one kind of site available for the hopping ions, hence all hops are geometrically alike.

(ii) After a hop, site relaxation is entirely due to the hopping motion of the other ions.

In a glass, assumption (i) is clearly not valid. In particular, the dynamic structure model [21] distinguishes between at least two kinds of sites in a single-alkali glass. These are the so-called $\bar{A}$ sites which are optimally configured to meet the requirements of each mobile $A$ ion (here: $\mathrm{Li}^{+}$), and the $\bar{C}$ sites which provide less room and are less favourable. If an $A$ ion leaves an $\bar{A}$ site, this site is supposed to stay $\bar{A}$ for some time and then to transform into a $\bar{C}$ site. On the other hand, if an $A$ ion hops into a $\bar{C}$ site and manages to stay, then the $\bar{C}$ site is supposed to relax into an $\bar{A}$ site.

Evidently, assumption (ii) is also invalid in a glass, since the local single-particle potential changes with time not only because of the hopping of the other ions and the shifting of the Coulomb cage, but also because of localized geometrical adjustments of the network itself. These adjustments could involve, for example, changes in $\mathrm{B}-\mathrm{O}-\mathrm{B}$ bond angles (in this borate glass) or, indeed, the making and breaking of $\mathrm{B}-\mathrm{O}$ bonds. Due to these processes, $\bar{A}$ and $\bar{C}$ sites can change their identity even below the glass transition temperature.

A logical extension of the dynamic structure model, in the light of the jump relaxation model, is to regard the $\bar{A}$ to $\bar{C}$ hops as being intrinsically as frequent as the $\bar{A}$ to $\bar{A}$ hops, but more easily reversed. The ion will simply roll back into its original $\bar{A}$ site, unless the $\bar{C}$ site changes to meet its requirements.

Invoking these new ideas, we are now able to replace the original jump relaxation model with a more complex scenario which properly accounts for the situation in a glass. In the following, some important features of this scenario will be read from the hoppingconductivity spectra of Figs. 3 to 6 .

\section{Power-Law Exponents}

In view of the spectra, two basic questions are:

(i) Why is $q>1$ possible and what does it mean?

(ii) Is there a physical criterion for the exponent in (4) to be smaller or larger than one? 


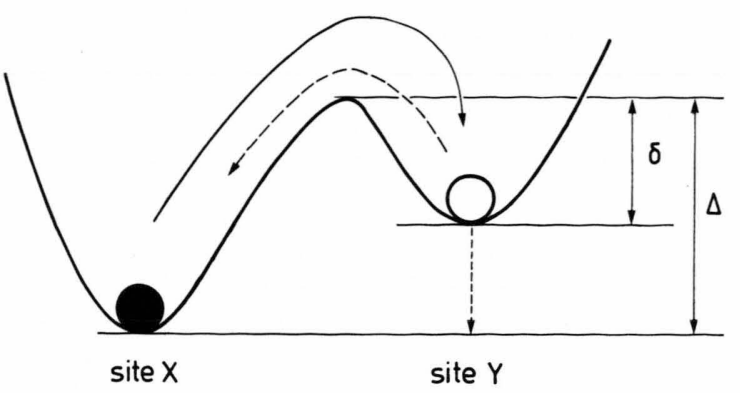

Fig. 10. Effective single-particle potential of the central ion at the time of its initial hop from site $X$ to site $Y$.

The answers to these questions are surprisingly simple, as will be shown in the following.

In Fig. 10, consider the situation just after a hop (to be called the initial hop) that an ion (the central ion) has performed from some site $X$ to some neighbouring site $Y$. Suppose the charge distribution and geometrical arrangement of its neighbourhood are both still relaxed with respect to $X$. At the time of the hop, the single-particle potential barriers from $X$ to $Y$ and from $Y$ to $X$ are $\Delta$ and $\delta$, respectively.

Once the ion is at $Y$, a competition sets in between two different ways of relaxation. The ion may either hop back to $X$, or the neighbourhood may relax with respect to $Y$.

The latter process of site relaxation may now include both charge relaxation, i.e. a shifting of the Coulomb cage by hops of other ions, and geometrical relaxation by network adjustment.

Either one of the two competing ways of relaxation can be characterized by a time constant or by its inverse, the respective rate. In the following, these rates are denoted by

$$
\begin{aligned}
& 1 / t^{*}:=\text { initial back-hop rate, } \\
& 1 / t_{1}:=\text { initial site-relaxation rate. }
\end{aligned}
$$

If $W(t)$ is the probability for the correlated backward hop from $Y$ to $X$ not to have occurred until time $t$ after the initial hop, then

$$
1 / t^{*}:=-\left(\frac{\mathrm{d} W(t)}{\mathrm{d} t}\right)_{t=0} .
$$

Likewise, if $\delta_{\text {back }}(t)$ is the time dependent barrier height for the backward hop from $Y$ to $X$, then

$$
1 / t_{1}:=\frac{1}{k T}\left(\frac{\mathrm{d} \delta_{\text {back }}(t)}{\mathrm{d} t}\right)_{t=0} .
$$

We will now show that questions (i) and (ii) can be answered simultaneously in terms of the rates $1 / t^{*}$ and $1 / t_{1}$. If the ratio of these rates, $t_{1} / t^{*}$, does not exceed two, then the relationship to the power-law exponent of the hopping conductivity is

power-law exponent

$$
=\frac{\text { initial back-hop rate }}{\text { initial site-relaxation rate }} \text {. }
$$

For larger values of $t_{1} / t^{*}$ the exponent stays at two.

While the assumptions of the original jump relaxation model imply that the ratio $p=t_{1} / t^{*}$ should always be smaller than one [7],

$$
\begin{aligned}
& p=1-\alpha, \\
& \alpha=\text { mismatch parameter with } 0<\alpha<1,
\end{aligned}
$$

typical scenarios with $q>1$ are easy to visualize if those assumptions are abandoned, see below.

In order to derive (10), let us start by considering the correlated back-hop rate, $-\dot{W}(t)=-\mathrm{d} W(t) / \mathrm{d} t$, at sufficiently short times, when the site-relaxation process at $Y$ is still far from being completed and when third hops of the central ion, from $X$ to $Y$ again, can still be neglected. Introducing an attempt frequency, $v_{0 Y}$, we then have

$$
-\dot{W}(t)=W(t) \cdot v_{0 Y} \cdot \exp \left[-\delta_{\text {back }}(t) / k T\right]
$$

or

$$
-\dot{W}(t) / W(t)=\left(1 / t^{*}\right) \cdot \exp \left[-\left(\delta_{\text {back }}(t)-\delta\right) / k T\right]
$$

with

$$
1 / t^{*}=v_{0 Y} \cdot \exp [-\delta / k T]
$$

Equations (12) and (13) are consistent with (8) as $W(0)$ is one and $\delta_{\text {back }}(0)$ is $\delta$. Of course, the shape of the normalized correlated back-hop rate, $-\dot{W}(t) / W(t)$, will strongly depend on the way $\delta_{\text {back }}$ develops with time. If there is no site relaxation at all, $\delta_{\text {back }} \equiv \delta$, then the normalized back-hop rate is constant, implying

$$
W(t)=\exp \left[-t / t^{*}\right]
$$

However, numerical simulations of the hopping of charged particles in disordered networks have shown [27] that the normalizied site-relaxational rate, $\dot{\delta}_{\text {back }}(t) / k T$, is at short times well described by

$$
\dot{\delta}_{\text {back }}(t) / k T=\frac{1}{t_{1}+t},
$$

which contains (9) as its $t \rightarrow 0$ limit. Equation (16) also holds within the framework of the jump relaxation model $[7,10,11]$. In fact, it had been derived from the 
coupled differential equations of that model before the above computer simulations were performed.

Integration of (16) yields a relation,

$$
\delta_{\text {back }}(t)-\delta=k T \cdot \ln \left(1+t / t_{1}\right),
$$

which is required for insertion into (13). Therefore, within the short-time regime, the normalized correlated back-hop rate is found to be

$$
-\dot{W}(t) / W(t)=\left(1 / t^{*}\right) \cdot\left[1 /\left(1+t / t_{1}\right)\right] .
$$

Equation (18) is now easily solved for $W(t)$, resulting in a power-law behaviour:

$$
W(t)=\left(1+t / t_{1}\right)^{-t_{1} / t^{*}} .
$$

Note that in the absence of any site relaxation, i.e. for $t_{1} \rightarrow \infty,(15)$ is recovered from (19):

$$
W(t)=\lim _{t_{1} \rightarrow \infty}\left(1+t / t_{1}\right)^{-t_{1} / t^{*}}=: \exp \left(-t / t^{*}\right) .
$$

We are now in a position that allows us to estimate the contribution to the high-frequency hopping conductivity which is due to the hopping between sites $X$ and $Y$ in Figure 10. In order to do so, we first relate the correlated back-hop rate to the (short-time part of the) velocity autocorrelation function describing hops between $X$ and $Y$ :

$$
\langle\boldsymbol{v}(0) \cdot \boldsymbol{v}(t)\rangle_{X, Y}=\left(x_{0}^{2} / 2\right) \cdot \Gamma_{0} \cdot[\delta(t)+\dot{W}(t)] .
$$

In (21), $\delta(t)$ is the delta function, while $x_{0}$ and $\Gamma_{0}$ are the $X-Y$ distance and the forward hopping rate, respectively:

$$
\Gamma_{0}=v_{0 X} \cdot \exp [-\Delta / k T] .
$$

Here $v_{0 X}$ denotes the attempt frequency at site $X$. To retain simplicity, we suppose that the influence of cross terms, $\left\langle\boldsymbol{v}_{i}(0) \cdot \boldsymbol{v}_{j}(t)\right\rangle$, where $i$ and $j$, with $i \neq j$, denote different hopping ions, can be neglected. The contribution to the high-frequency hopping conductivity that is due to the hopping between sites $X$ and $Y$ is then proportional to the Fourier transform of the velocity autocorrelation function of (21):

$$
\sigma_{X, Y}(\omega)=\sigma_{X, Y}(\infty) \cdot\left[1+\int_{0}^{\infty} \dot{W}(t) \cdot \cos (\omega t) \mathrm{d} t\right] .
$$

The power law of (19) now transforms into a power law of $\sigma_{X, Y}(\omega)$ :

(i) If the ratio $t_{1} / t^{*}$ is smaller than two, we have approximately

$$
\sigma_{X, Y}(\omega)=\sigma_{X, Y}(\infty) \cdot\left[1+\left(\omega t_{1}\right)^{-1}\right]^{-t_{1} / t^{*}} .
$$

More precisely, the Fourier transformation of (23) yields a result close to that of (24), with $\omega$ replaced by $\omega \cdot k\left(t_{1} / t^{*}\right)$, where

$$
k\left(t_{1} / t^{*}\right)=\left[\cos \left(\frac{\pi t_{1}}{2 t^{*}}\right) \cdot \frac{\Gamma\left(2-t_{1} / t^{*}\right)}{1-t_{1} / t^{*}}\right]^{t^{*} / t_{1}} .
$$

In (25), $\Gamma$ denotes the Gamma function. As $k$ is normally close to one, this factor is omitted in our equations, although it is taken into account in our numerical fits.

(ii) If $t_{1} / t^{*}$ tends to infinity, $\sigma_{X, Y}(\omega)$ is particularly simple:

$$
\sigma_{X, Y}(\omega)=\sigma_{X, Y}(\infty) \cdot\left[1+\left(\omega t^{*}\right)^{-2}\right]^{-1} .
$$

In this case, $\sigma_{X, Y}$ displays a power law with exponent two before merging into the high-frequency plateau.

(iii) The power-law exponent is, therefore, two for both limiting cases, $t_{1} / t^{*} \rightarrow 2$ and $t_{1} / t^{*} \rightarrow \infty$, the straight lines in a $\log \sigma$ vs. $\log \omega$ plot being parallel to each other (for given $t^{*}$ ). As might have been expected, finite values of $t_{1} / t^{*}$ larger than two yield results in between, again with an exponent of two.

Summarizing (i) to (iii) one can say that (10) does, indeed, hold if the ratio of $t_{1} / t^{*}$ does not exceed two. If it does, the exponent stays at two.

\section{Spectra and Hopping Processes}

Different cases may now be discussed. Two particularly important ones are those were

(i) $X$ is an $\bar{A}$ site and $Y$ is an $\bar{A}$ site,

(ii) $X$ is an $\bar{A}$ site and $Y$ is a $\bar{C}$ site.

Certainly, only a fraction of all mobile ions have a vacant $\bar{A}$ site in their immediate neighbourhood, while neighbouring $\bar{C}$ sites are rather more common. The $\bar{A}$ to $\bar{A}$ hops play an important role as the dc conductivity results essentially from an itinerant hopping motion of the mobile ions via $\bar{A}$ sites. These are in effect the conduction pathways. On the other hand, due to the larger abundance of $\bar{C}$ sites, the $\bar{A}$ to $\bar{C}$ hops might well provide a larger contribution to the high-frequency-plateau value of the conductivity than do the $\bar{A}$ to $\bar{A}$ hops.

Figure 11 is a sketch of typical single-particle potentials encountered by hopping ions (at $t=0$ ) in $\bar{A}$ to $\bar{A}$ and $\bar{A}$ to $\bar{C}$ hops. In either case, the effective total 
a)

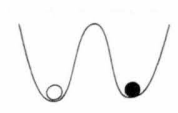

$+$
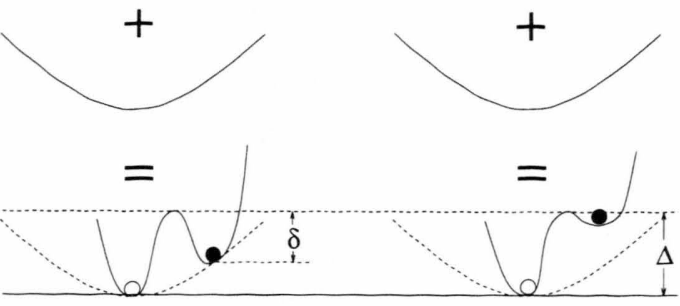

$\overline{\mathrm{A}} \quad \overline{\mathrm{A}}$

$\overline{\mathrm{A}} \quad \overline{\mathrm{C}}$

Fig. 11. Typical single-particle potentials encountered by hopping ions (at $t=0$ ) in a) $\bar{A}$ to $\bar{A}$ and b) $\bar{A}$ to $\bar{C}$ hops. Each of the effective potentials constructed in a) and b) consists of two contributions: a site-sensitive potential (on top) and a Coulomb cage potential.

potential is constructed by superposition of a site sensitive potential and a Coulomb-cage potential, the latter being due to the arrangement of the other mobile ions in the glass. The shapes of the effective potentials of Figs. $11 \mathrm{a}$ and $\mathrm{b}$ differ from each other because site $\bar{C}$ is a priori less favoured than site $\bar{A}$. Although the forward-hopping barrier heights, $\Delta$, may be similar in both cases, the back-hop barrier heights, $\delta$, will be quite different, being small or negligible for $\bar{C}$ to $\bar{A}$ backward hops.

After a forward hop ( $\bar{A}$ to $\bar{A}$ or $\bar{A}$ to $\bar{C})$, site relaxation sets in. For the $\bar{A}$ to $\bar{A}$ hopping, the situation is, indeed, well described by the jump relaxation model, since both sites are geometrically equivalent and since site relaxation is essentially achieved by the shifting of the Coulomb cage, due to other $\bar{A}$ to $\bar{A}$ hops. Therefore, $t_{1} / t^{*}$ is smaller than one in this case and can be identified with the power-law exponent, $p<1$, of the $\bar{A}$ to $\bar{A}$ hopping contribution to the total conductivity. As translational motion is essentially via $\bar{A}$ sites, this power law will directly merge into the de conductivity, according to (1). Moreover, the total frequency dependence of the $\bar{A}$ to $\bar{A}$ hopping contribution to the conductivity is supposed to be well described by (6), resulting in the log-log plot of Figure 12. The parameter values that have been used in this plot have - among others - also been employed in the model construction yielding the solid lines in Figs. 5 and 6 , see below.

The situation is different for $\bar{A}$ to $\bar{C}$ hops. On a $\bar{C}$ site, site relaxation will be due to both Coulomb relax-

ation and geometrical network adjustment. Accordingly, $t_{1}$ will be rather shorter than on an $\bar{A}$ site. At the same time, however, $t^{*}$ will be even much shorter than on an $\bar{A}$ site, as the back-hop barrier height is now small or negligible. The second effect is supposed to overcompensate the first, yielding a $t_{1} / t^{*}$ ratio larger than one, which is now identified with the power-law exponent $q>1$.

In fact, an essential part of the high-frequency hopping conductivity of Figs. 5 and 6 is easily explained in terms of $\bar{A}$ to $\bar{C}$ hops, which are mostly unsuccessful. While the optimum value for $t_{1} / t^{*}$ is $q=1.3$, the forward-hopping barrier height, $\Delta$, is supposed to be the same as for $\bar{A}$ to $\bar{A}$ hops.

The preexponential factor turns out to be larger for $\bar{A}$ to $\bar{C}$ than for $\bar{A}$ to $\bar{A}$ hops, reflecting the larger abundance of $\bar{C}$ sites. We assume that only a very small fraction of $\bar{A}$ to $\bar{C}$ hops turns out to be successful, due to $\bar{C}$ to $\bar{A}$ site relaxation. Their contribution to the dc conductivity is certainly swamped by the $\bar{A}$ to $\bar{A}$ hopping and can, therefore, not be detected in the spectra.

The temperature dependence of the high-frequency hopping conductivity, see Fig. 8, suggests that different processes, with different activation energies, predominate at comparatively high and low temperatures. The high-temperature process, with activation energy $\Delta$, has already been identified as $\bar{A}-\bar{C}$ hopping motion. The other one has a similar value of $q$ but is characterized by much smaller values of its preexponential factor. As the preexponential factor contains

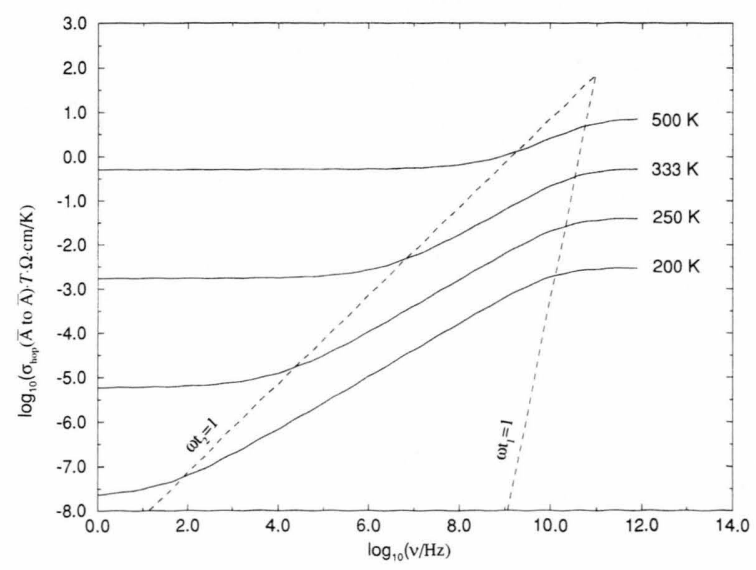

Fig. 12. Log-log representation of the calculated $\bar{A}$ to $\bar{A}$ hopping contribution to the frequency dependent conductivity of glassy $\mathrm{B}_{2} \mathrm{O}_{3}-0.56 \mathrm{Li}_{2} \mathrm{O}-0.45 \mathrm{LiBr}$, cf. (6). 
the square of the hopping distance, this process is easily viewed as being a small localized displacement or reorientation. This interpretation is in agreement with our previous discussion of high-frequency conductivities in glass $[19,28]$.

In summary, the model construction used to fit the data in Figs. 5, 6, and 8 takes account of

(i) the new universality, which provides a temperature independent baseline for $\sigma(\omega)$ as shown in Fig. 6,

(ii) the hopping of the mobile lithium ions via $\bar{A}$ sites, which is well described by the jump relaxation model, cf. Fig. 12,

(iii) the $\bar{A}$ to $\bar{C}$ hopping of the lithium ions, which is characterized by a ratio of $t_{1} / t^{*} \approx 1.3$ and is mostly unsuccessful, and

(iv) a localized small displacement or reorientation of ions within a given neighbourhood, featuring a similar ratio $t_{1} / t^{*}$.

For (ii) and (iii), the forward-hopping barrier height, $\Delta$, has been chosen to be $k \cdot 2600 \mathrm{~K}$ (about $0.22 \mathrm{eV}$ ), while $\delta$ is $k \cdot 550 \mathrm{~K}$ (about $0.05 \mathrm{eV}$ ) and zero, respectively. All attempt frequencies are close to $1 \mathrm{THz}$. - In (iv) we have used forward and backward activation energies of $k \cdot 480 \mathrm{~K}$ (about $0.04 \mathrm{eV}$ ) and zero, respectively. The power-law exponents are $p=0.6$ for (ii) and $q=1.3$ for (iii) and (iv).

With regard to the jump-relaxation part of the conductivity, cf. Fig. 12, it is also important to note that besides the activation energies our model calculation also makes use of Meyer-Neldel-type activation entropies [29-32], the ratio being a Meyer-Neldel temperature of $880 \mathrm{~K}$. This implies that the top of the triangular dispersive regime in Fig. 12 is at a value of $\log \left(\sigma_{\text {hop }}(\bar{A}\right.$ to $\left.\bar{A}) \cdot T\right)$ which is attained by extrapolation of $1 / T$ to the inverse Meyer-Neldel temperature, instead of zero. As a consequence, the dispersive regime of Fig. 12 now has a realistic width on the frequency scale, and there is no need for introducing unreasonably high attempt frequencies.

\section{Conclusion}

The presence of high-frequency plateaux in the hopping-conductivity spectra of ionically conducting glasses has now been firmly established. These $a c$ conductivity plateaux are readily identified once the contributions from vibrational motion (which are proportional to $\omega^{2}$ ) have been taken away from the total conductivity spectra.
This is a new result. It is important because the plateau marks the region where all ionic hopping processes are effective and contribute to the conductivity. By comparison, the application of electric fields at lower frequencies allows time during each cycle for the ions to return to their starting positions, and so it becomes apparent that many of the hops are unsuccessful. Only at much lower frequencies is the $d c$ plateau found, which is a measure of the much smaller number of successful hops which contribute to the long-range migration of ions.

Now that the high-frequency plateau has been located, it becomes possible to characterize the entire spectrum of hopping processes in glass. The most striking result is the identification of a new power law region, where $q>1$, which is seen just below the highfrequency plateau. At high temperature, the activation energy for the high-frequency plateau and the $q>1$ power-law region turns out to be quite similar to the one found in the more conventional low-frequency power-law regime, where the exponent is $p<1$. With decreasing temperatures, however, the activation energy for the plateau and $q>1$ regions changes to a significantly lower value.

For these diverse phenomena, a unified explanation is provided through a simple extension of the jump relaxation model. Apart from small localized reorientations which account for the low-energy high-frequency contribution, we propose that all hopping processes for a given mobile ion in glass require roughly the same energy and occur at roughly the same rate. The difference is that some hops are considered to be more easily reversed than others. These hops are therefore usually unsuccessful and make little or no contribution to the dc conductivity. They make their appearance only in the new power-law region and in the high-frequency plateau.

This seems to provide firm spectroscopic evidence for the existence of distinct $\bar{A}$ and $\bar{C}$ sites as indicated in the dynamic structure model of glass [21]. When a lithium ion, as in this instance, jumps into a $\bar{C}$ site, it does not find a suitable chemical environment for lithium ions, and so it is ready to return at the first opportunity into the lithium site ( $\bar{A}$ site) from which it came. Only if it stays in its new site long enough for relaxation of the local structure to occur, will this kind of hop be successful. On the other hand, if the ion jumps into a lithium site, the only reason for backward hops is the Coulombic field generated by the other mobile cations. 
The simple picture which emerges is that all successful ion hops in glass involve processes of environmental relaxation. If this relaxation involves mainly the motion of other mobile ions, this results in a dc plateau followed by a power law with $p<1$. If, however, chemical bonding and local structure are rearranged in the relaxation process, then a new power law appears with $q>1$. Such an interpretation is clearly at variance with any models of glass behaviour where the complexity of ion hopping processes would be attributed to static disorder within a random network structure.

[1] A. K. Jonscher, Nature London 267, 673 (1977).

[2] W.-K. Lee, J. F. Liu, and A. S. Nowick, Phys. Rev. Letters 67, 1559 (1991)

[3] A. S. Nowick, A. V. Vaysleyb, and B. S. Lim, J. NonCryst. Solids 172-174, 1243 (1994).

[4] R. Hoppe, T. Kloidt, and K. Funke, Ber. Bunsenges. Phys. Chem. 95, 1025 (1991).

[5] K. Funke, T. Kloidt, D. Wilmer, and C. J. Carlile, Solid State Ionics 53-56, 947 (1992).

[6] K. L. Ngai and U. Strom, Phys. Rev. B 38, 10350 (1988).

[7] K. Funke, Prog. Solid St. Chem. 22, 111 (1993).

[8] K. Funke and I. Riess, Z. Phys. Chem. NF 140, 217 (1984).

[9] K. Funke, Z. Phys. Chem. NF 154, 251 (1987).

[10] K. Funke, Mat. Res. Soc. Symp. Proc. 210, 97 (1991).

[11] K. Funke, Key Engng. Mat. 59, 60, 1 (1991).

[12] K. Funke, J. Non-Cryst. Solids 172-174, 1215 (1994).

[13] K. L. Ngai, Comm. Sol. State Phys. 9, 127 (1979).

[14] K. L. Ngai, Comm. Sol. State Phys. 9, 141 (1980).

[15] K. L. Ngai, S. L. Peng, and K. Y. Tseng, Physica A 191, 523 (1992).

[16] J. Wong and C. A. Angell, Glass, Structure by Spectroscopy, Dekker, New York, 1976.

[17] M. D. Ingram, Phys. Chem. Glasses 28, 215 (1987).

[18] A. Burns, G. D. Chryssikos, E. Tombari, R. H. Cole, and W. M. Risen Jr., Phys. Chem. Glasses 30, 264 (1989).

[19] C. Cramer, K. Funke, C. Vortkamp-Rückert, A. J. Dianoux, Physica A 191, 358 (1992).

\section{Acknowledgements}

It is a pleasure to thank A. Bunde for fruitful discussions. He contributed essential views to our present paper. The experimental and theoretical analysis of the frequency dependent ionic conductivity was funded by the Deutsche Forschungsgemeinschaft. Financial aid for our British-German collaboration is provided by the British Council and the DAAD in the framework of the ARC programme. Financial help by the Fonds der Chemischen Industrie is also gratefully acknowledged.

[20] A. Pradel and M. Ribes, J. Non-Cryst. Solids 172-174, 1315 (1994)

[21] A. Bunde, M. D. Ingram, P. Maass, J. Non-Cryst. Solids 172-174, 1222 (1994).

[22] A. Bunde, M. D. Ingram, P. Maass, and K. L. Ngai, J. Non-Cryst. Solids 131-133, 1109 (1991).

[23] A. Bunde, M. D. Ingram, P. Maass, K. L. Ngai, J. Phys. A24, L881 (1991).

[24] A. Bunde, P. Maass, and M. D. Ingram, Ber. Bunsenges. Phys. Chem. 95, 977 (1991).

[25] P. Maass, A. Bunde, and M. D. Ingram, Phys. Rev. Lett. 68, 3064 (1992).

[26] K. Funke, J. Kümpers, and J. Hermeling, Z. Naturforsch. 43a, 1094 (1988).

[27] P. Maass, J. Petersen, A. Bunde, W. Dieterich, and H. E. Roman, Phys. Rev. Lett. 66, 52 (1991).

[28] C. Cramer and K. Funke, Ber. Bunsenges. Phys. Chem. 96, 1725 (1992)

[29] W. Meyer and H. Neldel, Z. Techn. Physik 12, 588 (1937).

[30] W.-K. Lee, A. S. Nowick, and H. Jain, Solid State Ionics 28-30, 89 (1988)

[31] T. Dosdale and R. J. Brook, Solid State Ionics 18/19, 1105 (1986).

[32] D. P. Almond and A. R. West, Solid State Ionics 23, 27 (1987). 